\title{
FACHTEXTE-IN-VERNETZUNG ALS INTERDISZIPLINÄRER ZUGANG ZU FACHLICHEN KOMMUNIKATIONSRÄUMEN - EIN NEUES PARADIGMA DER AKTUELLEN FACHKOMMUNIKATIONSFORSCHUNG
}

\section{EINLEITUNG}

Unter dem Eindruck der seit den 1980er Jahre radikal zunehmenden Bedeutung der digitalen Informations- und Kommunikationsmedien (Internet, World Wide Web, E-Book u. a.) sieht sich die moderne Sprachwissenschaft veranlasst, die bisher dominante physische Interpretation des Verhältnisses von Sprache und Raum grundlegend zu überdenken (Bonfadelli/Jarren/Siegert 2010). Dabei hat sich ihre Hinwendung zur Kategorie des Raumes vor allem seit den 1990er Jahren deutlich verstärkt, obwohl unter dem Eindruck einiger zeitgenössischer Philosophen die Auffassung vertreten wurde, dass die frei kombinierbaren elektronischen Strukturen der Kommunikation (vgl. computertechnologische Telemedialität, News Groups, Mediennetzwerke u. a.) die direkten Zusammenhänge zwischen Raum und Kommunikation immer unbedeutender werden lassen (Rajewski 2002). So hat z. B. der französische Philosoph Paul Virilio (1986) hervorgehoben, dass die durch die elektronische Revolution bedingte angebliche „Ortlosigkeit“" der Kommunikation bzw. die „Deterritorialisierung“ von Sachverhalten und Prozessen der Realität zu einer „Ästhetik des Verschwindens" führt (vgl. Virilio 2008: 78). Die auch in anderen Wissenschaften vertretene These von der technologisch bedingten Irrelevanz des Raumes hat schließlich dazu geführt, dass gegenwärtig Wissenschaftsvertreter von unterschiedlichen erkenntnistheoretischen Positionen aus um ein modernes transdisziplinäres Raumkonzept ringen.

In der Linguistik macht die seit dem Ende der 1980er Jahre als topologische Wende bzw. spatial turn (Weigel 2013) bezeichnete Neuperspektivierung der Kategorie des Raums deutlich, dass eine interdisziplinär fundierte Konzeptualisierung der vielfältigen Sprache-Kommunikation-Raum-Beziehungen ansteht. Erste methodologisch-methodische Annäherungen an die realen Formen der Sprache-Raum-Relation sind hierbei z. B. die Analyse kartierbarer Räume von Sprachfamilien sowie Dialekten usw. durch die Sprachgeografie, Dialektologie bzw. areale Linguistik.

Aus unserer Sicht steht der Raumdiskurs bei einer umfassenden Darstellung der Sprache-Kommunikation-Raum-Beziehungen vor folgenden inhaltlichen Herausforderungen: 
1. Charakterisierung und Systematisierung der Vielfalt von (physikalischen, territorialen, gesellschaftlichen, kulturellen, ethnischen, virtuellen, kybernetischen u. a.) Räumen, Raumvorstellungen, Raumwahrnehmungen bzw. Raumverhältnissen als Voraussetzung für weiterführende Erkenntnisperspektiven,

2. Darstellung des Untersuchungsgegenstandes „Raum“ aus einer konkreten methodologisch-methodischen Perspektive (z. B. materialistisches vs. metaphysisches, absolutes vs. relationales, statisches vs. dynamisches bzw. geschlossenes vs. offenes Herangehen) (Läpple 1991: 157ff.),

3. Entwicklung von Strategien der konzeptionellen Bewältigung von Raum durch:

a) die Überlagerung physischer Räume durch soziale Räume (Netzwerke),

b) den Umgang mit sinnlich nicht wahrnehmbaren aber faktisch vorliegenden räumlichen Ordnungen (Telekommunikation),

c) die Bestätigung von Räumlichkeit durch die Präzisierung räumlicher Ordnungen (Logistik),

d) die Ablösung räumlicher durch funktionale Ordnungen (Funktionssysteme) u. a. (Stichweh 2008: 157),

5. Untersuchung der Spezifik der medientechnologisch bedingten Erweiterung des Raumkonzepts (z. B. Welche Räume können sich über welche Medien konstituieren?),

6. Analyse von kulturbezogenen bzw. einzelwissenschaftsspezifischen Raumvorstellungen (Raum als fachlich gebundenes Institutionalisierungsmuster: Sozialgeografie, Raumsoziologie, Geschichte, Theologie, Kartografie, Architektur, darstellende Künste, Musik, Literatur u. a.),

7. Umsetzung der territorialen, politischen, ökonomischen, ideologischen u. a. Globalisierungserfahrungen auf die Interpretation von Raum: z. B. Raum als Identitätsprinzip,

8. Einsatz von Raum als erkenntnistheoretisches Ordnungsprinzip z. B. bei der Strukturierung sozialer Interaktionsabläufe zwischen den an der Kommunikation Beteiligten oder beim Transfer von Datenmengen,

9. kognitionswissenschaftlich fundierte Erweiterung von Raum zum Wissenskonfigurations- bzw. Denkraum (vgl. mental mapping, images bzw. kognitive Landkarten: mentale Repräsentation von Abbildern der Realität; Bedeutung räumlicher Metaphern; Raum als Schema zur Erfassung der objektiven Realität; Textsortenspezifik von Denkräumen; Denkraum als dynamische Räumlichkeit; systematische Ausdifferenzierung von Denkräumen) (Hoffstadt 2009),

10. Konzipierung von Raum als a) Gefüge dynamischer Wechselbeziehungen kommunikativer Aktivitäten; b) infrastrukturelle Vernetzung globaler und lokaler elektronischer Medienensembles; c) Nutzungsgefüge kulturspezifischen, fachlichen u. a. Wissens; d) institutionelle Organisation von Kommunikationspartnern; e) Mehrdimensionalität von Kommunikation; f) Modus der Informationsgewinnung, Informationstransfer und Informationsnutzung; g) Mensch-Computer-Mensch Interaktivität u. a. (vgl. dazu Faßler 2001), 
11. Umsetzung eines konkreten Methodenpluralismus bei der empirischen Analyse von Raum unter Berücksichtigung strukturell-funktionaler, kommunikativ-kognitiver u. a. Zusammenhänge,

12. Differenzierung der konzeptuellen Verbindungen von (fachlicher) Intertextualität und (fachlichem) Kommunikationsraum (Hinwendung zu vielschichtigen (Fach-) Textsortennetzen) und

13. interdisziplinäre Modellierung des komplexen Zusammenhangs zwischen Mensch - (Fach-)Sprache - (Fach-)Kommunikation - (Fach-)Text(sortennetze) und Raum (d. h. Raum als Medium, das zwischen den Menschen (Fach-) Kommunikation vermittelt).

Im Unterschied zum Fachtext eröffnen Fachtexte-in-Vernetzung einen mehrdimensionalen Zugang zur Konstituierung fachlicher Kommunikationsräume. Sie machen unter wissenschaftsstrategischem Aspekt deutlich, dass der Raum eine grundlegende interaktive Existenzweise der Fachkommunikation im Allgemeinen und der Fachtexte-in-Vernetzung im Besonderen darstellt (Baumann 2008: 109ff.). Ein komplexes erkenntnistheoretisches Herangehen ermöglicht es, den fachlichen (Kommunikations-)Raum als eine Konstellation zu erfassen, die auf konkreten Relationen, Referenzen und/oder Konfigurationen zwischen den kommunikativ-kognitiven Determinationszusammenhängen der in der Fachkommunikation vorkommenden Fachtextsorten(netzen) beruhen.

Die Kategorie des Netzes ist durch ihre begriffliche Anschaulichkeit besonders geeignet, um weiterführende Erkenntnisse über die Breite, Tiefe, Dichte und Wechselwirkungen der raumbildenden Bezüge in der Fachkommunikation zu gewinnen (Döring/ Thielmann 2008).

Da Netzwerke die Eigenschaft aufweisen, Zusammenhangsmuster in der Interaktion der jeweiligen Partner modellieren zu können, entwickeln sie sich häufig zu Standards der Fachkommunikation.

\section{DIE KONZEPTE DER FACHLICHEN INTERTEXTUALITÄT UND DES RAUMS}

\subsection{Anfänge der Intertextualitätsforschung}

Seit dem Beginn der 1980er Jahre gebührt den beiden Textlinguisten R. de Beaugrande und W. Dressler (1981: 192ff.) das wissenschaftliche Verdienst, die wechselseitigen Beziehungen und Abhängigkeiten, die zwischen Texten bestehen, als ein konstitutives Kriterium von (fachlicher) Textualität erkannt zu haben. Sie heben die zentrale Bedeutung der Intertextualität für die Ausprägung von Textsorten folgendermaßen hervor: „Intertextualität ist, ganz allgemein, für die Entwicklung von TEXTSORTEN als Klassen von Texten mit typischen Mustern von Eigenschaften verantwortlich..." (ebd.: 13).

Im Weiteren haben R. de Beaugrande und W. Dressler deutlich gemacht, dass das Konzept der Intertextualität unverzichtbar ist, um „die Abhängigkeit zwischen Produktion bzw. Rezeption eines gegebenen Textes und dem Wissen der 
Kommunikationsteilnehmer über andere Texte" aufzuzeigen (ebd.: 188 - Hervorhebung im Zitat - K.-D. B.).

Mit diesem über den Gegenstandsbereich der Linguistik hinausgehenden Entwurf haben R. de Beaugrande und W. Dressler der Entwicklung interdisziplinärer Beschreibungsansätze im Allgemeinen und der komplexen Betrachtung von Kommunikationsraum im Besonderen den Weg bereitet.

\subsection{Typologisierung von Intertextualitätsansätzen}

Einer der ersten raumorientierten Intertextualitätsansätze hat sich zu Beginn der 1980er Jahre entwickelt. Das Modell der globalen Intertextualität stützt sich dabei auf einen erweiterten, sich selbst reproduzierenden offenen Textbegriff, welcher die Grenzen der Prätexte überschreitet und alle semantischen Strukturen unter den Begriff der Intertextualität subsumiert. Nach Auffassung von J. Kristeva (1972: 348) ist jedem Text globale Intertextualität eigen: „,...jeder Text baut sich als Mosaik von Zitaten auf, jeder Text ist Absorption und Transformation eines anderen Textes". Sie schlussfolgert daraus, dass kein Text isoliert zu betrachten ist. Damit konzipiert sie den universalen Intertext, der Räumliches integriert (vgl. Laiko 2004).

Auch die translatorische Intertextualität geht offensichtlich von einer Raumdeterminiertheit kommunikativer Handlungen aus, wobei das Fachtextmusterwissen von der Ausgangs- in die Zielsprache transferiert wird (Baumann 2009a). Das Modul des Räumlichen bezieht sich im Rahmen dieses Ansatzes auf die vom Dolmetscher bzw. Übersetzer vermittelten (teil)äquivalenten/nichtäquivalenten Beziehungen zwischen ausgangs- und zielsprachlichen Fachtext(sort)en sowie den personenvermittelten textbasierten Transfer zwischen mindestens zwei Einzelsprachen.

Darüber hinaus integrieren weitere Intertextualitätsmodelle die Perspektive des Räumlichen. Dazu zählen z. B. die:

1. interaktionale Intertextualität, die sich auf das ortsgebundene soziale Wechselverhältnis zwischen den erkennenden bzw. kommunizierenden Subjekten der Fachkommunikation und dem in Fachtexten transferierten Wissen bezieht (Baumann 1992; 1994; Wichter/Antos 2001);

2. kooperative Intertextualität, die durch (Fach-)Texte auf unmittelbar vorangegangene (Fach-)Texte reagiert, wobei nach Auffassung von L. Wilske und W.-D. Krause (1987: 894ff.), ,in der Regel das Ganze des vorangegangenen Textexemplars inhaltlich erfasst und die Textsortenqualität desselben verarbeitet", d. h. mit einer kooperativen (Fach-)Textsorte geantwortet wird (vgl. Nachricht - Dementi; Aufruf - Stellungnahme; Briefwechsel: Brief - Antwortbrief). Auch bei diesem Intertextualitätskonzept, das entscheidend zur Ausprägung von Fachtext(sort)enin Vernetzung beiträgt, spielt die räumliche Interaktion zwischen den Produzenten der betreffenden Fachtexte eine herausragende Rolle (Krause 2000: 63ff.);

3. kommunikationssituationsbezogene Intertextualität analysiert den Einfluss der raumdeterminierten sozialen, Tätigkeits- bzw. Umgebungssituation der Kommunikationspartner auf die Vernetzung von Fachtext(sort)en (Baumann 2001; Müller 2006); 
4. textmediale Intertextualität geht den spezifischen Auswirkungen der mündlichen, schriftlichen, elektronischen Kommunikationsmedien auf die Konstituierung räumlich organisierter Fachtextvernetzungen (Hypertexte) nach (Hufeisen/Marx 2004);

5. bimediale Text-Bild-Intertextualität tritt besonders im Kommunikationsbereich der Werbung auf. Bilder sind bekanntlich oft mehrdeutig. (Fach-)Texte haben die Aufgabe, die Mehrdeutigkeit der Bilder für den Adressaten einzuschränken und die Interpretation zu präzisieren. Die Dimensionen von Text und Bild müssen sich aufeinander beziehen und sich ergänzen, um ein integratives Gesamtverständnis zu erreichen. S.-P. Ballstaedt (1997) unterscheidet drei Arten von raumbezogenen Text-Bild-Beziehungen:

a. kongruente Bezüge: Der Text beschreibt, was das Bild zeigt,

b. komplementäre Bezüge: Der Text hat Leerstellen, die das Bild ausfüllt (und umgekehrt) sowie

c. elaborative Bezüge: Der Text geht über die Bildinhalte hinaus (und umgekehrt) (Hanna 2003; Takayama-Wichter 2005: 203ff.);

6. kognitionsbezogene Intertextualität untersucht:

a. die vielschichtigen Zusammenhänge zwischen kognitiver und kommunikativer Tätigkeit - d. h. zwischen Denken, Wissen und den räumlich determinierten Interaktionsmustern in der Fachkommunikation - und

b. die Bedeutung der bei den Kommunikationspartnern implizit/explizit vorhandenen Fachwissensmuster (vgl. schema/frame/script: Bartlett 1958) für die mehrdimensionale Fachtextvernetzung (Baumann 2001).

7. funktionale, generische, referentielle Intertextualität als differenzierte Zugänge zum vielschichtigen funktionalen und interaktionalen Beziehungsgeflecht, das zwischen entsprechenden Fachtextsorten besteht und konkrete Rückschlüsse auf deren sozial-institutionelles Beziehungsgefüge zulässt (vgl. Steuerbuchhaltung) (Devitt 1991: 337);

8. typologische Intertextualität bezieht sich u. a. auf die Spezifik der Raumbeziehungen, die zwischen den einzelnen Fachtextexemplaren und den übergreifenden Fachtextsortenmustern bestehen. Sie manifestiert sich dort, wo die intertextuellen Relationen auf funktionalen und strukturellen Übereinstimmungen zwischen Fachtexten beruhen, da sie für das Erkennen von Fachtextmustern und die Klassifizierung von Fachtextsorten bedeutsam sind (z. B. Fachtextbauplan von naturwissenschaftlichen Zeitschriftenartikeln: Introduction, Method, Results, Discussion; Standardisierung von Fachtextmustern im Bereich der Technik, stabilisierte/ritualisierte Fachtextanfänge, z. B. in der juristischen Fachkommunikation) (Petöfi/Olivi 1988; Holthuis 1993: 34);

9. Fachtextsorten-in-Relation als eine Form von Intertextualität existiert nur im Zusammenhang mit einer Trägerfachtextsorte (z. B. Vorwort bei Monografien und Lehrbüchern; wissenschaftliches Werk bei Rezensionen). Die Trägerfachtextsorte kann hingegen auch ohne die Fachtextsorte-in-Relation existieren. Zwischen diesen beiden Fachtextarten besteht folglich ein asymmetrisches Abhängigkeitsverhältnis (Timm 1996: 458ff.) und 
10. rhetorische Intertextualität, die davon ausgeht, dass die durch Tropen markierten raumbezogenen Bezüge der Fachtextvernetzung zur Ausprägung einer bestimmten (historischen) Atmosphäre beitragen bzw. die soziolektale Vielfalt der Fachkommunikation gestalten (Lachmann 1990).

Schließlich sind erste interdisziplinär orientierte Herangehensweisen zu der Erkenntnis gelangt, dass fachliche Intertextualität ein kommunikativ-kognitives Phänomen darstellt und auf dieser Grundlage die Produktion und Rezeption von Fachtexten als wissensbasierte Prozesse auch räumlich miteinander verbunden sind, wobei Raum hierbei als ein Modell von Zusammenhängen verstanden wird (Faßler 2008: 192ff.; Jablonski/Rau/Ritzke 1990; Hillert 1990; Baumann 1992, 2001; Rickheit/Strohner 1993; Felix/Habel/Rickheit 1994; Habel/Kanngießer/Rickheit 1996; Preyer/Ulkan/Ulfig 1997).

In diesem Umfeld ist zweifellos ein gewaltiges Erkenntnispotential angelegt, das z. B. die raumbezogenen Aspekte der sprachlichen Exteriorisierung und Interiorisierung fachwissenschaftlicher Kenntnisse und die damit verbundenen Strategien des kommunikativen Transfers von mentalen Abbildern der fachlichen Realität ebenso einschließt wie die sprachlichen bzw. nichtsprachlichen Referenzmechanismen der sich konstituierenden Fachtexte, Fachtextsorten bzw. Fachtextsortennetze (Holthuis 1993: 114ff.).

Damit rücken vor allem die folgenden zwei Aufgabenkomplexe in den Mittelpunkt entsprechender Untersuchungen:

1. die umfassende Analyse der sprachlichen bzw. nichtsprachlichen Differenzierungsmechanismen fachlicher Intertextualität als erster Schritt, um die Strukturierung fachkommunikativer Räume detailliert erfassen zu können und

2. die Darstellung von Raum als komplexe Konstituierungsgröße von Fachtext(sort)en-in-Vernetzung.

Aus wissenschaftsstrategischer Perspektive führt dieses Herangehen zu einer radikalen Erweiterung des fachkommunikativen Untersuchungsbereiches, die sich darin äußert, dass die gegenwärtige Fachsprachenlinguistik beginnt, nicht mehr ausschließlich den Fachtext oder die Fachtextsorte als Manifestationen von Kommunikationsräumen zu betrachten, sondern vor allem Fachtext(sorten)-in-Vernetzung.

\section{DIE INTERDISZIPLINÄRE BETRACHTUNG VON FACHLICHEN KOM- MUNIKATIONSRÄUMEN}

\subsection{Fachtexte als Fachtextsorten-in-Vernetzung}

Die auf der Grundlage interdisziplinär fundierter Fachtextbeschreibungsmodelle erfolgte methodologisch-methodische Sichtung der fachlichen Intertextualitätsansätze hat gezeigt, dass das Konzept der Fachtextsorten-in-Vernetzung und die Konstituierung fachlicher Kommunikationsräume dialektisch miteinander in folgender Hinsicht verbunden sind (Baumann 1992; 1994; 2001):

1. Das Phänomen der Fachtext(sort)en-in-Vernetzung ist ein Hinweis auf die Entstehung fachlicher Kommunikationsräume, in denen es bekanntlich um den optimalen Transfer fachbezogener Informationen zwischen Fachleuten und Fachleuten 
(horizontale Wissensvermittlung) bzw. Fachleuten und Nichtfachleuten (vertikale Wissensvermittlung) geht. Das sich hieraus ergebende dynamische prozessuale Kommunikationsraumverständnis hat weit reichende methodologische Auswirkungen auf die Auffassung von Fachtexten als Fachtext(sort)en-in-Vernetzung (Baumann 1992: 104ff.; Schweigkofler 2007).

2. Fachtext(sort)en-in-Vernetzung verbinden die Produktion und Rezeption von Fachtexten ebenso miteinander wie den damit verbundenen Aufbau von Fachwissen, dessen kommunikative Umsetzung und kommunikationsräumliche Verankerung.

Ausgehend davon werden folgende methodologische Positionen impliziert, die den fachlichen Kommunikationsraum betreffen:

2.1. Fachtext(sort)en-in-Vernetzung setzen die intensive/extensive Interaktion von mindestens zwei Kommunikationspartnern voraus.

2.2. Fachtext(sort)en-in- Vernetzung stellen das Ergebnis einer Vielzahl ineinander greifender kommunikativ-kognitiver Prozesse dar.

2.3. Fachtext(sort)en-in-Vernetzung ist Prozess und Resultat zugleich: Sie beruhen auf den Prozessen der Instrumentalisierung und Operationalisierung unterschiedlicher Kenntnissysteme, deren vielschichtiges Zusammenwirken durch Referenzbezüge hinsichtlich des fachgegenständlichen Inhaltsbezugs, der sprachlichen Form, der kommunikativen Funktion bzw. der räumlichen Dimension der Fachtext(sort)en-in-Vernetzung deutlich wird.

2.4. Die Betrachtung der Fachtext(sort)en-in-Vernetzung impliziert mehrere Untersuchungsrichtungen:

2.4.1. die Analyse strukturell-funktionaler, kommunikativ-kognitiver Bezugsmechanismen der einzelnen Fachtexte (,intratextuelle“ Intertextualitätsanalyse),

2.4.2. die Darstellung strukturell-funktionaler, kommunikativ-kognitiver Bezugsmechanismen zwischen Fachtexten einer Fachtextsorte (,intertextuelle" Phase der fachlichen Intertextualitätsanalyse) und

2.4.3. die Darlegung strukturell-funktionaler, kommunikativ-kognitiver Bezugsmechanismen zwischen Fachtexten mehrerer Fachtextsorten („Vernetzungsphase“ der fachlichen Intertextualitätsanalyse) (Baumann 2001).

3. Sowohl induktiv-empirische Fachtextanalysen (Kalverkämper/Baumann 1996; Baumann/Kalverkämper 2004; Baumann 2009b: 197ff.) als auch neurowissenschaftliche Untersuchungen von Fachkommunikation (Kochendörfer 1999; Pauen/Roth 2001; Wildgen 2008 u. a.) weisen darauf hin, dass die Fachtext(sort) en-in-Vernetzung das Ergebnis hochkomplexer strukturell-funktionaler, kommunikativ-kognitiver Prozesse darstellen, die darauf gerichtet sind, das jeweilige Fachwissen in modularisierter Form in die Fachkommunikation zu integrieren. Mit diesem Vorgehen eröffnet sich ein historisch-konkreter Zugang zu den durch die Scientific Community in fachlichen Kommunikationsräumen gespeicherten Fachwissensbeständen. 
4. Das Konzept der Fachtext(sort)en-in-Vernetzung setzt bei den Produzenten und Rezipienten von Fachtexten metalinguistische Kenntnisse darüber voraus, welche prototypischen Relationsmuster bzw. Vernetzungsregularitäten von Fachtext(sort)en in welchen konkreten Handlungsräumen, Wissenschaftsgebieten und Einzelsprachen tradiert sind (vgl. z. B. Journalismus: referentielle Relationen zwischen der vorangegangenen Fachtextsorte Zeitungsbericht und der nachfolgenden Fachtextsorte Kommentar, die sich beide auf ein und die selbe Nachricht beziehen) (Beaugrande/Dressler 1981: 13; Kalverkämper 1981).

5. Die vielschichtige Vernetzung von Fachtext(sort)en manifestiert sich in einem komplexen Gesamtprozess sowohl produktiver als auch rezeptiver Phasen, der auf strukturell-funktionalen, kommunikativ-kognitiven, räumlichen und zeitlichen Mechanismen beruht, die dazu beitragen, einen historisch-konkreten Fachwissensstand als Ergebnis des Gedankenaustausches zwischen fachlich Handelnden sprachlich adäquat im Kommunikationsprozess umzusetzen. Dabei werden entsprechende Fachdenkstrategien, Fachwissenskontexte und Fachwissensbestände in fachlichen Kommunikationsräumen miteinander verbunden. Aus dieser Komplexität von Bezügen heraus ergibt sich eine Vielzahl von Beschreibungsdimensionen fachlicher Kommunikationsräume, die nur auf interdisziplinärer Grundlage erfolgreich erforscht werden können.

Im Weiteren wollen wir die wissenschaftlichen Bezugsebenen des interdisziplinären Konzepts der fachlichen Kommunikationsräume in deszendenter Reihenfolge vorstellen.

\subsection{DIMENSIONEN DER FACHLICHEN KOMMUNIKATIONSRÄUME}

\subsubsection{Die Raumdimension der Kultur(en)}

Infolge der sich seit den 1990er Jahren vollziehenden Globalisierung aller gesellschaftlichen Beziehungen hat sich die interdisziplinäre Fachsprachenforschung verstärkt der kulturellen Gebundenheit von Fachtext(sort)en-in-Vernetzung zugewandt (Kalverkämper 1988; Baumann 2001). So konnte in zahlreichen kontrastiven Fachtextanalysen nachgewiesen werden, dass die Fachkommunikation in konkreten Wissenschaften, Wissenskontexten, Situationen, Interaktionen, Einzelsprachen bzw. zwischen mehreren (nicht-) fachlichen Kommunikationsgemeinschaften kulturspezifischen Einflüssen unterliegt, die sich z. B. in Form kulturell determinierter Vernetzungsstrategien von Fachtext(sort)en als fachliche Kommunikationsräume manifestieren (Kalverkämper 1996: 683ff.). In diesem Kontext erweisen sich kulturspezifische Fachtextvernetzungsstandards von großer analytischer Bedeutung. Darunter verstehen wir als Erstes eine komplexe, empirisch fundierte Beschreibungsgröße für die Fachtextkonstitution in einer konkreten Kultur aus dem Blickwinkel einer anderen Kultur (vgl. Clyne 1993: 3-18; Thomas 2000: 231-279; Knapp 2004: 409-430).

Als Zweites erfassen wir unter kulturspezifischen Fachtextvernetzungsstandards tradierte dynamische Vertextungsregularitäten von Fachtext(sort)en, denen kulturell 
bestimmte Normen und Konventionen bezüglich der Produktion bzw. Rezeption von Fachtext(sort)en zugrunde liegen. Die Nichtbeachtung oder das Überschreiten eines bestimmten Toleranzbereiches von kulturspezifischen Fachtextvernetzungsstandards führt zwangsläufig zu Störungen bei der Konstituierung fachlicher Kommunikationsräume bzw. zum Abbruch des fachlichen Kommunikationsprozesses.

Dabei sind kulturspezifische Fachtextvernetzungsstandards als hierarchisch strukturierte Phänomene angelegt, die auf empirisch abgesicherten Klassifikationsebenen die Realisierung kultureller Faktoren einbeziehen.

Erste empirische Untersuchungen weisen darauf hin, dass es wechselseitige Zusammenhänge zwischen kulturspezifischen Fachtextvernetzungsstandards einerseits sowie der strukturell-funktionalen bzw. räumlichen Ausprägung/sozialen Qualität/Art der Handlungsorientiertheit von Fachtext(sort)en-in-Vernetzung andererseits gibt (Baumann 2008: 109ff.).

Weiteren Untersuchungen bleibt es vorbehalten, die raumkonstituierenden Erkenntnisse über das kulturelle Determinationsgefüge von Fachtext(sorten)netzen zu erweitern.

\subsubsection{Die Raumdimension der Sozialität}

Die Integration der an der Fachkommunikation Beteiligten in ein bestimmtes Gesellschaftssystem spielt bekanntlich für die Verlaufsqualität und sozio-räumliche Verankerung der Fachkommunikation eine Schlüsselrolle. Aus dieser Einsicht ergibt sich das anhaltende Bemühen, die innere Dynamik der historisch konkreten Gesellschaftsstruktur in Beziehung zur Struktur und Funktion der Fachkommunikation in verschiedenen Wissenschaftsbereichen bzw. fachlichen Kommunikationsräumen zu untersuchen (Baumann 1992). So haben vor allem Fachtextanalysen in den 1980er Jahren darauf hingewiesen, dass außer der fachlichen Tätigkeitssituation, in welcher die Kommunikationspartner Fachtexte bzw. Fachtextsorten zueinander in Beziehung setzen (vgl. Konstellation der Kommunikationspartner, historisch konkretes Entwicklungsniveau und Entwicklungstempo der betreffenden Fachwissenschaft), auch die soziale Situation (vgl. sozialer Status der Kommunikationspartner, Wertvorstellungen, soziale Normen, Gewohnheiten, Denkmuster der Partner, Bekanntheitsgrad und soziale Nähe bzw. Distanz der Kommunikationspartner) bzw. die Umgebungssituation der beteiligten Kommunikationspartner (Qualität der zwischenmenschlichen Beziehungen, Grad der inhaltlichen und sprachlichen Kompetenz der Kommunikationspartner, Alter, Geschlecht, Ort u. a.) für interdisziplinäre Vertextungsanalysen unter sozialen Gesichtspunkten aufschlussreich sind (Baumann 1992: 170ff.).

Folglich stellen die konkrete Sozialität der Kommunikationspartner sowie der konkrete soziale Charakter ihrer Beziehungen untereinander erkenntnistheoretisch bedeutsame Faktoren der gesellschaftlichen Determinierung von Fachtext(sort)en-in-Vernetzung bzw. der Konfiguration von Kommunikationsräumen dar. Aktuelle Untersuchungen zur sozialen Markiertheit fachlicher Intertextualität bzw. fachlicher Kommunikationsräume konzentrieren sich darauf, die Bedeutung nachfolgender Aspekte und Relationen zu klären: 
1. das Verhältnis zwischen der sozialen Vernetzung von Gruppen(mitgliedern) [(Nicht-) Fachleute], der Vernetzung von Fachtexten bzw. Fachtextsorten (vgl. z. B. Fachtextsorte Berufs-Bewerbung im Deutschen und Französischen) und der Konstituierung fachlicher Kommunikationsräume (Morello 2006),

2. die Korrelation zwischen verschiedenen Formen der praktischen und theoretischen Zusammenarbeit von (fachintern, interfachlich bzw. fachextern agierenden) Angehörigen der Scientific Community, der Ausprägung von mehr oder weniger stabilen sozialen Beziehungsnetzwerken, der Bildung von charakteristischen Fachtext(sort) en-in-Vernetzung bzw. fachlicher Kommunikationsräume (Faßler 2001),

3. die Dynamik des Wechselverhältnisses zwischen Beziehungsnetzwerken der an der Fachkommunikation Beteiligten in den Gesellschafts-, Natur- bzw. Technikwissenschaften und der Ausprägung spezifischer Fachtext(sorten)netze in konkreten fachlichen Kommunikationsräumen (Baumann 2009c, 13-36),

4. die Charakterisierung der Sozialität von Fachleuten und Nichtfachleuten, des gesellschaftlichen Charakters ihrer kommunikativen Beziehungen sowie der Ausbildung fachlicher Intertextualität/fachlicher Kommunikationsräume (Osolnik Kunc 2006) und

5. die Ermittlung derjenigen sozialen Faktoren, die für die Konventionalisierung von Fachtext(sort)en-in-Vernetzung/fachlichen Kommunikationsräumen signifikant sind (Keil 2007).

Die hierbei zu erwartenden Analyseergebnisse werden dazu beitragen, die soziale Basis der konkreten Bezüge zwischen Fachtexten bzw. Fachtextsorten und fachlichem Kommunikationsraum genauer zu erfassen.

\subsubsection{Die Raumdimension der Situativität}

Bekanntlich ist jede (mündliche/schriftliche) Fachkommunikation an eine bestimmte externe bzw. interne Situation der Beteiligten gebunden. Während sich die externe Situation auf die Vielzahl der ökonomischen, sozialen, politischen, ideologischen, räumlichen Determinanten von Kommunikation bezieht (vgl. auch 2. Die Bezugsebene der Sozialität), beschäftigt sich die interne Situation mit der inneren Einstellung, (affektiven/ kognitiven) Verfasstheit, Motivation(sstärke), Quantität und Qualität von individuellen Fachwissensbeständen, dem Urteilsvermögen, den Wertvorstellungen bzw. kognitiven Reaktionen von den an der Fachkommunikation beteiligten Partnern (Knoblauch 2005).

Im Mittelpunkt der Situativitätsanalyse steht demzufolge die Frage, in welcher Weise die an der Fachkommunikation Beteiligten die Aspekte der konkreten externen Situation durch Elemente der internen Situation reflektieren. Bei der Erörterung der komplexen Korrelation zwischen externer und interner Situation können wir uns bereits auf erste Erkenntnisse von Untersuchungen der fachlichen Intertextualität stützen (Baumann 2008: 118ff.): So spiegelt sich z. B. der Aspekt der Situativität der an der Fachkommunikation Beteiligten in solchen Makrostruktureinheiten von Fachtext(sort)en wider, deren Funktion darin besteht, die konkrete Einstellung des Fachtextautors zum antizipierten Vorwissensbestand des Partners in der Fachtextrezeption zu signalisieren. 
$\mathrm{Zu}$ diesen die innere Einstellung des Fachtextautors vermittelnden intertextuellen Einheiten gehören z. B. das für einige Fachtextsorten obligatorische Vorwort (Prolog, Einführung), der Aufgabenteil bei didaktisch aufbereiteten Fachtextsorten bzw. die Teiltexte, in denen bestimmte affektive/kognitive Aspekte der Interaktionsbeziehungen der Beteiligten aktualisiert werden (Befehl, Forderung, Anweisung u. a.).

Die Faktoren der internen Situation sind für die fachliche Intertextualität besonders dort relevant, wo es - bedingt durch die entsprechenden Erwartungen der Kommunikationspartner - um (in Zeit und Raum) verbindlich geregelte Handlungsabläufe und die daraus folgenden weitgehend konventionalisierten Fachtextvernetzungen geht (Traueranzeige - Nekrolog von Wissenschaftlern; Auszeichnung - Laudatio von Fachleuten usw.).

In weiterführenden Untersuchungen gilt es aufzuzeigen, auf welche Weise die vielschichtigen Interdependenzen der externen und internen Situation auf die spezifische Vernetzung von Fachtext(sort)en und fachlichen Kommunikationsräumen Einfluss nehmen.

\subsubsection{Die Raumdimension der Intermedialität}

Die intermedialen Bezüge zwischen schöngeistiger Literatur und Film werden bekanntlich bereits seit der Entstehung des Mediums Film gegen Ende des 19. Jahrhunderts diskutiert. Allerdings beginnt die gezielte Erörterung des methodologischen Stellenwerts, den intermediale Bezüge für die Erforschung der literarischen Intertextualität einnehmen, erst seit den 1970er Jahren (Rajewski 2002: 29ff.). Schließlich wird Intermedialität in den 1980er und 1990er Jahren vor allem von Literaturwissenschaftlern und Linguisten als kommunikativ-semiotisches Konzept verstanden, das wesentlich zur intertextuellen Bedeutungskonstituierung beiträgt.

In den 1990er Jahren beginnen auch die Vertreter der Fachsprachenforschung die grundlegende methodologisch-methodische Bedeutung der Intermedialität für die integrative Betrachtung der Fachkommunikation zu erkennen (Schröder 1993; Mayer 1998). Empirische fachsprachenlinguistische Untersuchungen weisen darauf hin, dass Fachtexte in den elektronischen Medien aus textstruktureller Sicht enger miteinander verbunden sind als Fachtexte in den Printmedien (Raasch/Kühlwein 1984; Hess-Lüttich/Holly/Püschel 1996). Zudem wird deutlich, dass infolge der Intermedialität größere Variationsräume für Vernetzungen von Fachtext(sort)en entstehen (Internetportale) (Wiese 2004: 121-127; Opilowski 2005; Ostapenko 2007).

Seit einigen Jahren wird die Kategorie des Hypertextes für die interdisziplinäre Betrachtung der fachlichen Intertextualität bzw. Intermedialität vor allem im Bereich der fachlichen Werbung als viel versprechend angesehen. Unter einem Hypertext ist eine nicht-lineare Organisation von Objekten zu verstehen, deren netzartige Struktur durch logische Verbindungen (Hyperlinks) zwischen Wissenseinheiten (Knoten: z. B. Texte oder Textteile) hergestellt wird. Der Hypertext beruht auf der Anwendung des so genannten Verweis-Knoten-Konzepts ${ }^{1}$ (Hufeisen/Marx 2004).

1 Ein Knoten in einem Hypertext ist aus Sicht der Informationswissenschaft die entscheidende Wissenseinheit. Diese fungiert als Anfangs- und Endpunkt jener logischen Verbindungen, welche durch Hyperlinks (Verweis-Knoten-Verbindung) hergestellt werden. 
Gegenwärtig besteht in der interdisziplinären Fachsprachenforschung das Desiderat eines die strukturell-funktionale bzw. semiotische Vielfalt intermedialer Bezüge widerspiegelnden Entwurfs, der sich auf eine repräsentative Anzahl von kommunikationsraumevozierenden Fachtext(sort)en-in-Vernetzung aus verschiedenen Einzelsprachen, Einzelwissenschaften, Handlungszusammenhängen und Medien stützt. Im Kontext intermedialer Bezüge und ihrer Bedeutung für die Konstituierung fachlicher Intertextualität ergeben sich mehrere Problemstellungen:

1. Welche erkenntnistheoretischen Beziehungen bestehen zwischen den Phänomenen der fachlichen Intertextualität und Intermedialität?

2. Welche Analysekategorien sind auch räumlich abgrenz- und handhabbar, um die intermediale Bezugnahme auf die Fachkommunikation adäquat zu erfassen?

3. Gibt es in diesem Zusammenhang Intermedialitätssignale (vgl. z. B. die Erarbeitung von E-Learning Plattformen für den fremdsprachlichen Fachsprachenunterricht)?

4. Wie lassen sich raumevozierende intra- und intermediale Bezüge in Fachtext(sorten)netzen kommunikativ realisieren (z. B. Kopplung von fachlichem Bild und Fachtext) (Kalverkämper 1996: 683ff.; Rajewski 2002: 69ff.)?

5. Wie konstituiert sich eine Fachtext(sorte)-in-Vernetzung in Relation zu anderen medialen (semiotischen) Systemen?

6. Welches methodische Analyseinstrumentarium ist hierfür geeignet?

7. Welche Auswirkungen haben die vielfältigen intermedialen Bezüge (Medienwechsel/Medienkombination) auf die Funktionalität des fachlichen Informationstransfers, die Konstituierung von Fachtext(sort)en-in-Vernetzung bzw. die Konfiguration fachlicher Kommunikationsräume (vgl. z. B. im Kommunikationsbereich Medizin die diagnostische Auswertung bildgebender Verfahren und deren kommunikative Umsetzung in Fachtextsortennetzen wie Epikrise, Befund, Überweisung usw.)?

8. Inwieweit bestimmen die Auswahl bzw. Vertextung fachlicher Gegenstände und Prozesse der Geistes-, Natur- und Technikwissenschaften die Einbeziehung bestimmter medialer (semiotischer) Systeme in die entsprechenden Fachtexte/ Fachtextsorten/fachlichen Kommunikationsräume?

Zweifellos bedarf es noch mehrerer intermedialer Fachkommunikationsanalysen, um das komplexe Verhältnis zwischen fachlicher Intertextualität, Raumbezogenheit bzw. Intermedialität aus interdisziplinärer Sicht befriedigend klären zu können.

\subsubsection{Die Raumdimension des Fachdenkens}

In interdisziplinären Analysen zur fachlichen Intertextualität nimmt die Kategorie des Fachdenkens eine zentrale erkenntnistheoretische Position ein (Baumann 1992: 144ff.), da sie darauf gerichtet ist, die Besonderheiten des Erkenntnisprozesses in einem konkreten fachlich begrenzbaren Bereich der Wirklichkeit systematisch zu erfassen bzw. zu einem Denkraum zu verorten (Hoffstadt 2009: 5ff.). Das Fachdenken wird somit als ein besonders komplexer kognitiver Prozess verstanden, der auf einem interpretierenden bzw. Ordnung stiftenden Verarbeiten von Informationen beruht 
(Vogler 2006, 2008; Keil 2007; Kühn 2007; Baumann 2009b: 197-222; Vogler 2009: 223-256).

Bisherige Fachtextanalysen aus verschiedenen Wissenschaften und Einzelsprachen zeigen, dass die konkreten Strategien des Fachdenkens einen geeigneten methodologisch-methodischen Ausgangspunkt für die Betrachtung des Sprachtransfers von begrifflich fixierten Abbildern der Fachinhalte darstellen (Baumann 2001). Ein um die Aspekte der fachlichen Intertextualität erweitertes Herangehen zielt nunmehr darauf ab, eine Typologie von Strategien des Fachdenkens zu entwickeln, auf deren Grundlage dann ein System von intertextuellen Regularitäten abgeleitet werden kann, das bei der Umsetzung von Abbildern der fachlichen Realität zwischen Fachtext(sort)en auftritt und zur „Verräumlichung“ von Fachwissen beiträgt.

Bei diesem ganzheitlichen Vorgehen besteht ein erster Schritt fachlicher Intertextualitätsuntersuchungen darin, die Vielfalt der einzelnen Wissenschaftsdisziplinen unter dem Aspekt der kognitiven Operationen, Strategien und Verlaufsqualitäten der geistigen Tätigkeiten zu beschreiben, welche die Art und Weise bestimmen, wie sich die Fachleute in ihren fachlichen Kommunikationsräumen orientieren und aufeinander beziehen (Faßler 2001). In einem zweiten Erkenntnisschritt wird die vielschichtige kommunikative Umsetzung der fachlichen Denkweise betrachtet, die durch weitgehend konventionalisierte Vernetzungen von Fachtext(sort)en untersetzt wird. Ein dritter Schwerpunkt richtet sich darauf, die gedanklichen Abbilder der Klassen von Inhalten, mit denen im jeweiligen Fach operiert wird sowie die sich in fachspezifischen Tätigkeitszusammenhängen ergebenden Denkleistungen in eine dialektische Verbindung zu bringen und diese dann bis zu deren kommunikativen bzw. semiotischen Manifestation in Fachtext(sorten)vernetzungen und fachlichen Kommunikationsräumen weiter zu verfolgen.

Damit bezieht sich dieser letzte Analyseschritt u. a. auf die räumliche Repräsentation von Fachwissen in Fachtext(sorten)netzen (Schäfer 2010).

Ein Teil unserer aktuellen Forschungskapazitäten konzentriert sich darauf, fachliche Intertextualität im Zusammenhang mit der Konstituierung von Fachwissen zu charakterisieren: Zum einen weist die Vernetzung von Fachtext(sort)en auf die ihr zugrunde liegenden verbundenen Wissensverortungen (Wissenskontexte) hin, zum anderen wird damit der Weg für die Generierung qualitativ neuen Fachwissens bereitet.

\subsubsection{Die Raumdimension des Fachinhalts}

Interdisziplinäre Fachtextuntersuchungen machen deutlich, dass die wissenschaftliche Differenzierung der fachbezogenen Realität mit einer strukturell-funktionalen Differenzierung fachlicher Intertextualität verbunden ist (Baumann 1994). Aus wissenschaftsstrategischer Sicht ist es demzufolge erforderlich, den konkreten Einfluss zu ermitteln, den der jeweilige fachliche Gegenstand auf die unterschiedlichen Vernetzungsstrategien und -muster von Fachtext(sort)en bzw. Konstituierung von fachspezifischen Kommunikationsräumen ausübt.

Unter methodologischen Gesichtspunkten gehen wir in unseren empirischen Analysen von der dialektischen Einheit zwischen Einheitlichkeit und Differenziertheit 
der Fachsprache aus (Hoffmann 1984: 53ff; Hoffmann/Kalverkämper/Wiegand 1998, 1999). Sie zielen darauf ab, eine strukturell-funktionale Verwandtschaft von Fachsprachen aufzuzeigen, die an Wissenschaften mit vergleichbaren Objektbereichen (Natur, Gesellschaft, Denken u. a.) gebunden ist.

Im Hinblick auf den Grad der Einheitlichkeit bzw. Differenziertheit der einzelnen Fachwissenschaften und ihrer Fachsprachen werden im Allgemeinen drei Gliederungskomplexe unterschieden:

1. Fachsprachen der Naturwissenschaften (vgl. exakte und biologische Naturwissenschaften),

2. Fachsprachen der Gesellschaftswissenschaften (Philosophie, Kultur- und Sozialwissenschaften, Politikwissenschaften, Wirtschafts- und Rechtswissenschaften, Sprach- und Kunstwissenschaften, Pädagogik, Ethnologie, Anthropologie u. a.) und

3. Fachsprachen der Technikwissenschaften (Verfahrenstechnik, Medizintechnik, Maschinenbautechnik, Kerntechnik, Biotechnik, Umwelttechnik, Verkehrstechnik, Bergbautechnik u. a.).

Aktuelle wissenschaftstheoretische Darstellungen sind derzeit bemüht, das inhaltlich-gegenständliche Selbstverständnis der Fächer als inhaltlich spezifischen Kommunikationsraum, die Komplexität des Fachdenkens sowie die Differenziertheit der Fachsprachen in den Natur-, Gesellschafts- und Technikwissenschaften im Verbund herauszuarbeiten (Baumann 2009d: 2241-2257). Ein Schwerpunkt dieser komplexen Betrachtungen ist darauf gerichtet, die erkenntnistheoretischen Grundnormen, Grundwerte und räumlichen Perspektiven des fachbezogenen Handelns systematisch zu dokumentieren. Dabei wird in den Darlegungen vor allem der Frage nachgegangen, wie fachliche Intertextualität angelegt ist oder sein muss, um die Erkenntnisgewinnung und den Informationstransfer in den einzelnen Wissenschafts- und Kommunikationsräumen zu optimieren.

Erste komparative Analysen verschiedener Fachtext(sorten)netze aus unterschiedlichen Einzelwissenschaften und Einzelsprachen akzentuieren vor allem drei Determinanten, welche die Tragweite der inhaltsbezogenen Ebene bei der Auswahl und Verwendung konkreter Vernetzungsmuster von Fachtext(sort)en unterstreichen:

a) Der Einfluss der Einzelwissenschaft ist entscheidend, denn intertextuelle raumevozierende Bezugsmuster von Fachtext(sort)en sind einzelwissenschaftlich determiniert, z. B. auf den Gebieten von Journalismus/Medienwissenschaften: Leitartikel - Kommentar, Nachricht - Dementi sowie in der Medizin: Überweisung - Anamnese - Befund - Epikrise u. a.

b) Das Verhältnis der an der Fachkommunikation Beteiligten zum Gegenstand beeinflusst die kommunikative Realisierung der fachlichen Intertextualität (z. B. Bildtext in den Fachtext(sort)en der Architektur, Kraftfahrzeugtechnik u. a.), aktiviert bei der konkreten raumbezogenen Umsetzung der fachlichen Intertextualität wichtige erkenntnisfördernde Impulse (Rationalität/Emotionalität), welche die Auseinandersetzung des Partners mit dem Fachtextinhalt modifizieren (Baumann 2008: 109ff.) und 
c) die Korrelation von inhaltlich-gegenständlicher Systematik im Fachtext und den individuellen Leistungsdispositionen der an der Fachkommunikation Beteiligten bestimmt die kompetente Auswahl der räumlichen Vernetzungsmuster von Fachtext(sort)en (Vorwort, Anmerkungen, Glossar in Fachtexten) (Busch/ Stenschke 2004).

\subsubsection{Die Raumdimension der Funktion}

Nach wie vor stellt die funktionale Typologisierung der Zusammenhänge zwischen Fachtext(sorten)netzen ein Desiderat der interdisziplinären Fachsprachenforschung dar. Dabei wollen wir im Rahmen dieses Analysekontexts unter der Funktion fachlicher Intertextualität die kommunikative Absicht verstehen, die der Produzent von Fachtext(sort)en-in-Vernetzung in konkreten (fachlichen) Räumen des Informationstransfers gegenüber dem/den anvisierten Rezipienten dieser Fachtext(sorten)vernetzungen verfolgt und kommunikativ umsetzt.

Von maßgeblicher Bedeutung für die funktionale Analyse fachlicher Intertextualität ist dabei zweifellos die funktional-kommunikative Betrachtung der zwischen den Fachtext(sort)en bestehenden Vernetzungsbeziehungen. Dabei werden zwei hierarchisch gestufte Untersuchungsebenen unterschieden, die jeweils für die innertextuelle bzw. außertextuelle Dimension der funktionalen Aspekte fachlicher Intertextualität stehen:

a) die intratextuelle Perspektive des Kommunikationsraums, die darauf gerichtet ist, die fachtext(sorten)spezifische Zusammensetzung von kommunikativen Äußerungsmustern innerhalb von Fachtext(sort)en aufzuzeigen (z. B. Fachtextsorte „Bedienungsanleitung“ als spezifisches Vernetzungsmuster mit den gedanklich-sprachlichen Operationen Anweisen, Empfehlen, Fordern als typische Fachtextbausteine) (vgl. Konzept der Kommunikationsverfahren: Schmidt $\left.1981^{2}\right)$ und - aufbauend darauf -

b) die intertextuelle Perspektive des Kommunikationsraums, die dazu beiträgt, dass Fachtextsortenvernetzungsstrategien eruiert werden können, die - im Interesse eines effektiven Fachinformationstransfers - komplexe kommunikative Handlung(styp) en umsetzen (Informieren, Aktivieren, Klären, Kontaktieren) (Schröder 2003).

Beide Beschreibungsstufen stellen eine anwendbare Typologisierungsbasis für die Funktionalität von Fachtext(sorten)netzen und fachlichen Kommunikationsräumen dar (Baumann 1992: 104ff.; Heinemann/Heinemann 2002: 188ff.; Adamzik 2004).

\subsubsection{Die Raumdimension der Fachtext(sorten)strukturen}

In Darlegungen zur fachlichen Intertextualität wird zu Recht davon ausgegangen, dass Fachtextvernetzungen als Basiseinheiten der Kommunikationsverortung anzusehen

2 Die genannten geistig-sprachlichen Operationen tragen als Kommunikationsverfahren entscheidend zum Vernetzen von Fachtexten bei, da sie auf Faktoren des Kommunikationsprozesses rekurrieren, die mit der Analyse bzw. Synthese begrifflicher Vorstellungen, dem Aufdecken von Relationen zwischen Elementen und dem Anweisen zukünftigen Handelns zusammenhängen (Michel 1985). 
sind (Diatlova 2003). Sie tragen entscheidend dazu bei, die strukturellen Elemente und Relationen unserer (inneren und äußeren) fachlichen Tätigkeiten zusammenhängend widerzuspiegeln (Leont'ev/Leont'ev/Judin 1984: 19ff.).

Entsprechende empirische Analysen weisen darauf hin, dass bei der kommunikativen Gestaltung des Fachwissenstransfers typische raumevozierende Vernetzungsregularitäten von Fachtext(sort)en beobachtet werden können (wissenschaftlicher Zeitschriftenartikel - Leserzuschrift; Monografie - Rezension; Stellenausschreibung - Bewerbungsschreiben; Gesetzbuch - juristische Urteilsfassung; Vorvertrag - Vertrag; Nachricht - Kommentar; Kundenerhebung - Werbeanzeige - Bestellung - Rechnung, Abstract - wissenschaftlicher Zeitschriftenartikel u. a.), die bekanntlich in struktureller und funktionaler Hinsicht stark normiert sind (Baumann 2005: 32-47). In diesem Zusammenhang darf nicht außer Acht gelassen werden, dass die Kohärenz der Fachtextsortennetze offensichtlich mit fachlogischen Gliederungsmodellen von Sachproblemen verbunden ist (z. B. bei: Gebrauchsanweisung, Kochrezept, medizinische Kasuistik, Grünbuch/Weißbuch in der EU-Politik) (Klauke 1993 u. a.).

Fachtext(sorten)strukturelle Vernetzungsregularitäten tragen dazu bei, die Ökonomie und Präzision des Informationsaustausches zwischen den Kommunikationspartnern zu gewährleisten. Darüber hinaus sind die konventionalisierten Fachtextverbindungen darauf gerichtet, den intertextuellen Fachtextrezeptionsprozess durch erwartbare Strukturen zu erleichtern, bestimmte Algorithmen zur Inhaltserschließung von Fachtext(sort)en zu etablieren sowie fachliches Handeln im Kommunikationsraum verbindlich zu regeln.

Demzufolge besteht ein Desiderat fachtextsortenstruktureller Intertextualitätsanalysen darin, die folgenden methodologischen Schwerpunkte systematisch zu analysieren:

a) Welches Gefüge von strukturellen Elementen und Relationen trägt zur Vernetzung von Fachtext(sort)en bei?

b) Welche Konsequenzen hat das Konzept der strukturellen Intertextualität für die aktuellen Fachtextklassifikationsmodelle (Krause 2000; Swales 2004)?

c) Welche erkenntnistheoretischen Weiterungen impliziert die Hinwendung der interdisziplinären Fachsprachenforschung zu Fachtextsortennetzen und fachlichen Kommunikationsräumen (Klein/Fix 1997)?

d) Welche Abstufungen von struktureller Intertextualität und Räumlichkeit können an Fachtextsortennetzen unterschieden werden (Hess-Lüttich 1997: 130ff.)?

e) Wie verhalten sich Intertextualitäts- bzw. Fachlichkeitsgrade und die Enstehung fachlicher Kommunikationsräume zueinander (Baumann 1994)?

f) Welche strukturellen Aspekte sind für die lineare Reihung bzw. hierarchische Vernetzung von Fachtext(sort)en kennzeichnend?

g) Welche strukturellen Charakteristika bestehen bei einer Einfachvernetzung bzw. Vielfachvernetzung (Einbettung) von Fachtext(sort)en (Klein 2000: 34ff.; Opilowski 2005)?

h) Wie werden implizite vs. explizite Bezüge zwischen den linearen bzw. hierarchischen Zusammenhängen von Fachtext(sort)en realisiert? 
i) Inwieweit wird die Entwicklung fachlicher Kommunikationsräume durch Vernetzungen von Fachtextsorten und konkrete Wissensbestände der an der Kommunikation Beteiligten beeinflusst (Baumann 2001)?

j) $\mathrm{Zu}$ welchen methodologischen Veränderungen des Raumkonzepts wird der Ansatz der strukturellen Intertextualität von Fachtext(sort)en in der (Angewandten) Linguistik führen?

Die nun folgenden - teilweise bereits eingehend untersuchten - Bezugsebenen des interdisziplinären Konzepts fachlicher Intertextualität beziehen sich allesamt auf sprachliche Aspekte der Vernetzung von Fachtext(sort)en:

\subsubsection{Die Raumdimension der Syntax und Morphologie}

Sie umfasst diejenigen syntaktischen bzw. morphologischen Elemente, die sich auf die Vermittlung von Visualität und Perspektive zwischen Fachtexten beziehen. $\mathrm{Zu}$ diesen gehören z. B. folgende Kategorien: Tempusfolge, Thematisierung und Rhematisierung, Ellipsenbildung, Bedeutungsexpansion und -kondensation, lineare Anordnung einzelner Textkonstituenten, texteröffnende und -schließende syntaktische Fertigstücke in bestimmten Fachtextsorten (z. B. des Rechts), anaphorische und kataphorische Textkonstituenten, Referenzstrukturen (Proformen, Deiktika, Indefinita, Nomina propria und Appellativa) und Prädikationsstrukturen von Fachtexten, die Verschränkung von Fachtext und Bildtext, parataktische und hypotaktische Satzkonfigurationen und Mittel der Satzverflechtungen, Redeformen (Monolog, Dialog, Polylog) und Wiedergabe fremder Rede (Zitate) sowie textkonstituierende Funktionen der Wortstellung u. a.

\subsubsection{Die Raumdimension des Stils}

Die stilistische Ebene der fachlichen Intertextualität spielt bei der Gestaltung räumlicher Vorstellungen in Fachtext(sorten)netzen eine bedeutende konstitutive Rolle.

So sind stilistisch relevante Elemente darauf gerichtet, komplexe Kommunikationsräume zwischen den vernetzten Fachtext(sort)en zu gestalten (vgl. z. B. Verwendung von Raum-, Orientierungs- und Bewegungsmetaphern).

Repräsentative Untersuchungen haben gezeigt, dass die stilistische Ebene der fachlichen Intertextualität vor allem durch die den Kommunikationsbedingungen adäquate Wahl von Ausdrucksvarianten fachliche Kommunikationsräume auszeichnet (Baumann 1992: 47ff.). Durch die Systematisierung und Klassifizierung stilistisch relevanter Erscheinungen konnte nachgewiesen werden, dass spezifische Stilelemente besonders geeignet sind, effizient zur Perspektivierung der Vernetzungsvielfalt von Fachtext(sort)en beizutragen (z. B. Emphase, Anapher und Parallelismus, Antithese, expandierte Metapher, Wiederholung) und somit eine konkrete Stilebene zu realisieren.

So trifft der Fachtextproduzent eine Auswahl aus den Stilmitteln, welche geeignet sind, eine konkrete Funktion der mehrdimensionalen Fachtextvernetzungen 
umzusetzen. Dies betrifft z. B. die Verwendung von Zitaten, die Entfaltung des Fachtextthemas auf der Grundlage einer bestimmten Kompositionsprinzipien folgenden Gedankenführung, den Grad der sprachlich expliziten Wiedergabe sachlogischer Zusammenhänge bzw. die Variation verschiedener lexikalischer Elemente.

\subsubsection{Die Raumdimension der Lexik und Semantik}

Die gegenstandsadäquate Auswahl und Verwendungsweise lexikalisch-semantischer Einheiten in der Fachkommunikation stellen eine grundlegende Dimension der fachlichen Intertextualität und der Ausprägung fachlicher Kommunikationsräume dar.

Die Terminologie als Kern des Fachwortschatzes spiegelt bei der Fachtextvernetzung eine erkenntnistheoretisch zentrale Rolle (Schippan 1984). Da das terminologische System der Systematik der betreffenden Fachwissenschaft folgt und den höchsten Grad der begrifflichen Abstraktion verkörpert, tragen Termini zur Konstituierung eines sehr hoch fachlichen Kommunikationsraums, einer optimalen Verständigung zwischen den Fachleuten und somit zur lexikalisch-semantischen Fundierung der fachlichen Intertextualität bei.

\subsubsection{Die Raumdimension der Grafik}

Sie konzentriert sich auf das Layout des Fachtextes, das Schriftbild, die typographischen Besonderheiten als Faktoren der Fachtextvernetzung bzw. Verortung von Fachkommunikation (Vignetten, Initialen, Einrückungen, Kursivierung, Zitate, Verhältnis von Fachtext und Fußnote, Bildtext) (Hübler 2001).

\subsubsection{Die Raumdimension der Orthographie}

Diese Ebene ist erkenntnistheoretisch viel versprechend für eine interdisziplinäre Betrachtung des Konzepts der fachlichen Intertextualität und des Raums, da sie für die Perspektivierung der schriftlichen Fachkommunikation unerlässlich ist. Sie besitzt den höchsten Grad an gesellschaftlicher Verbindlichkeit und trägt präskriptiven Charakter (Duden 2004). Groß- und Kleinschreibung, Interpunktion u. a. machen so die räumliche Ordnung der Fachkommunikation sichtbar.

\subsubsection{Die Raumdimension der Phonetik und Phonologie}

Sie bindet jede Form der mündlichen Fachkommunikation an bestimmte Ausspracheregeln bzw. rhythmische Strukturen (z. B. Assonanz, Alliteration, Reimbildung) und trägt somit zur Intertextualität mündlicher Fachtexte bei.

\section{SCHLUSS}

Die vorliegende Betrachtung fachlicher Intertextualität und Kommunikationsräume hat deutlich gemacht, dass sie das Ergebnis eines außerordentlich komplexen Zusammenspiels unterschiedlicher Bereiche der Fachkommunikation darstellen. Die Spezifik der fachlichen Intertextualität als Zugang zu fachlichen Kommunikationsräumen stützt sich dabei auf ein vielschichtiges Beziehungsgeflecht der aufgezeigten Bezugsebenen: 
Fachtexte bzw. Fachtextsorten kristallisieren sich in diesem Zusammenhang als komplexe Schnittpunkte vieler Fachtexte und Fachtextsorten heraus.

Zukünftigen Untersuchungen bleibt es vorbehalten, das skizzierte interdisziplinäre Modell fachlicher Intertextualität und fachlicher Kommunikationsräume durch empirische Analysen und theoretische Überlegungen auf den einzelnen Bezugsebenen weiter zu untermauern.

\section{Literatur}

ADAMZIK, Kirsten (Hrsg.) (2000) Textsorten: Reflexionen und Analysen. Tübingen: Stauffenburg.

ADAMZIK, Kirsten (2004) Textlinguistik: Eine einführende Darstellung. Tübingen: Niemeyer.

AGRICOLA, Erhard (1979) Textstruktur, Textanalyse, Informationskern. Leipzig: Verlag Enzyklopädie.

BARTLETT, Frederic C. (1958) Thinking: An experimental and social study. London: Allen and Unwin.

BALLSTAEDT, Steffen-Peter (1997) Wissensvermittlung: Die Gestaltung von Lernmaterial. Weinheim: Beltz Psychologische VerlagsUnion.

BAUMANN, Klaus-Dieter (1992) Integrative Fachtextlinguistik. Tübingen: Gunter Narr.

BAUMANN, Klaus-Dieter (1994) Fachlichkeit von Texten. Egelsbach/Frankfurt (Main)/Washington: Hänsel-Hohenhausen.

BAUMANN, Klaus-Dieter (2001) Kenntnissysteme im Fachtext. Egelsbach/Frankfurt a. Main/München/New York: Hänsel-Hohenhausen.

BAUMANN, Klaus-Dieter (2005) „Das komplexe Normensystem der Fachkommunikation." Fachsprache 1-2, 27, 32-47.

BAUMANN, Klaus-Dieter (2008) „Fachtexte-in-Vernetzung aus interdisziplinärer Sicht.“ In: I. Barz/U. Fix (Hrsg.), Fachtextsorten - Gestern und Heute. Frankfurt a. Main/Berlin/Bern: Peter Lang, 109-127.

BAUMANN, Klaus-Dieter (2008) „Die interdisziplinären Grundlagen vergleichender Analysen der Verwaltungssprache.“ In: E. Tabares Plasencia/V. Ivanova/E. Krüger (Hrsg.), Analisis linguistico contrastivo de textos especializados en espanol y deman. Berlin: Frank \& Timme Verlag, 31-43.

BAUMANN, Klaus-Dieter (Hrsg.) (2009a) Translatologie aus integrativer Sicht. Übersetzungswissenschaftliche Analysen zwischen System und Globalität. Hamburg: Dr. Kovac.

BAUMANN, Klaus-Dieter (2009b) „Die Ermittlung von Strategien des Fachdenkens in der Fachkommunikation von Natur- und Technikwissenschaften“. In: K.-D. Baumann (Hrsg.), 197-222.

BAUMANN, Klaus-Dieter (2009c) "A communicative-cognitive analysis of the information transfer in English and German LSP texts." In: St. Cavagnoli/E. Di Giovanni/R. Merlini (Hrsg.), La ricerca nella communicazione interlinguistica. Modelli teorici et metodologici. Milano: FrancoAngeli, 13-36. 
BAUMANN, Klaus-Dieter (2009d) „135. Sprache in Naturwissenschaften und Technik.“ In: U. Fix/A. Gardt/J. Knape (Hrsg.), Rhetorik und Stilistik. Ein internationales Handbuch historischer und systematischer Forschung. 2. Halbband. Berlin/ New York: Walter de Gruyter, 2241-2257.

BAUMANN, Klaus-Dieter/Hartwig KALVERKÄMPER (Hrsg.) (2004) Pluralität in der Fachsprachenforschung. Tübingen: Gunter Narr.

BEAUGRANDE, Robert-Alain de/Wolfgang Ulrich DRESSLER (1981) Einführung in die Textlinguistik. Tübingen: Niemeyer.

BONFADELLI, Heinz/Otfried JARREN/Gabriele SIEGERT (Hrsg.) (2010) Einführung in die Publizistikwissenschaft. Stuttgart: UTB.

BUSCH, Albert/Oliver STENSCHKE (Hrsg.) (2004) Wissenstransfer und gesellschaftliche Kommunikation. Frankfurt a. Main: Peter Lang.

CLYNE, Michael (1993) „Pragmatik, Textstruktur und kulturelle Werte. Eine interkulturelle Perspektive.“ In: H. Schröder (Hrsg.), Fachtextpragmatik. Tübingen: Gunter Narr. 3-18.

DEVITT, Amy J. (1991) „Intertextuality in Tax Accounting: Generic, Referential and Functional." In: C. Bazermann//J. Paradis (Hrsg.), Textual dynamics of the Professions: Historical and Contemporary Studies of Writing in Professional Communities. Madison: University of Wisconson Press. 336-357.

DIATLOVA, Irina (2003) Unternehmenstexte: Textsorten, Textcluster, topische Muster. Frankfurt a. Main: Peter Lang.

DÖRING, Jörg/Tristan THIELMANN (2008) Spatial Turn: Das Raumparadigma in den Kultur- und Sozialwissenschaften. Bielefeld: Transcript Verlag.

DUDEN (2004) Die deutsche Rechtschreibung. 23. Auflage. Band 1. Mannheim/Leipzig/Wien/Zürich: Dudenverlag.

FAßLER, Manfred (2001) Netzwerke: Einführung in die Netzstrukturen, Netzkulturen und verteilte Gesellschaftlichkeit. München: Fink.

FELIX, Sascha W./Christopher HABEL/Gert RICHHEIT (Hrsg.) (1994) Kognitive Linguistik. Repräsentation und Prozesse. Opladen: Westdeutscher Verlag.

HABEL, Christopher/Siegfried KANNGIEßER/Gert RICKHEIT (Hrsg.) (1996) Perspektiven der kognitiven Linguistik. Modelle und Methoden. Opladen: Westdeutscher Verlag.

HANNA, Ortrun (2003) Wissensvermittlung durch Sprache und Bild. Frankfurt a. Main: Peter Lang.

HEINEMANN, Margot/Wolfgang HEINEMANN (2002) Grundlagen der Textlinguistik: Interaktion - Text-Diskurs. Tübingen: Niemeyer.

HESS-LÜTTICH, Ernest W.B./Werner HOLLY/Ulrich PÜSCHEL (Hrsg.) (1996) Textstrukturen im Medienwandel. Frankfurt a. Main: Peter Lang.

HESS-LÜTTICH, Ernest W.B (1997) Text, Intertext, Hypertext - Zur Texttheorie der Hypertextualität. In: J. Klein/U. Fix (Hrsg.), 125-148.

HILLERT, Dieter (1990) Sprachprozesse und Wissensstrukturen: Neuropsychologische Grundlagen der Kognition. Opladen: Westdeutscher Verlag. 
HOFFMANN, Lothar (1984) Kommunikationsmittel Fachsprache: Eine Einführung. 2., überarb. Auflage. Berlin: Akademie Verlag.

HOFFMANN, Lothar/Hartwig KALVERKÄMPER/Herbert Ernst WIEGAND (Hrsg.) (1998) Fachsprachen. Languages for Special Purposes. Ein internationales Handbuch zur Fachsprachenforschung und Terminologiewissenschaft. 1. Halbband. Berlin/New York: Walter de Gruyter.

HOFFMANN, Lothar/Hartwig KALVERKÄMPER/Herbert Ernst WIEGAND (Hrsg.) (1999) Fachsprachen. Languages for Special Purposes. Ein internationales Handbuch zur Fachsprachenforschung und Terminologiewissenschaft. 2. Halbband. Berlin/New York: Walter de Gruyter.

HOFFSTADT, Christian F. (2009) „Denkräume“ und „Denkbewegungen “: Untersuchungen zum metaphorischen Gebrauch der Sprache der Räumlichkeit. Karlsruhe: Niemeyer.

HÜBLER, Axel (2001) Das Konzept ,Körper' in den Sprach- und Kommunikationswissenschaften. Tübingen/Basel: A. Francke.

HUFEISEN, Britta/Nicole MARX (Hrsg.) (2004) Beim Schwedischlernen sind Englisch und Deutsch ganz hilfsvoll: Untersuchungen zum multiplen Sprachenlernen. Frankfurt a. Main: Peter Lang.

JABLONSKI, Konrad/Armin RAU/Johannes RITZKE (1990) Wissensbasierte Textgenerierung. Tübingen: Gunter Narr.

KALVERKÄMPER, Hartwig (1981) Orientierung zur Textlinguistik. Tübingen: Niemeyer.

KALVERKÄMPER, Hartwig (Hrsg.) (1988) Fachsprachen in der Romania. Tübingen: Gunter Narr.

KALVERKÄMPER, Hartwig (1996) „Die Kultur des literarischen wissenschaftlichen Dialogs - aufgezeigt an einem Beispiel aus der italienischen Renaissance (Galilei) und der französischen Aufklärung (Fontenelle).“ In: H. Kalverkämper/K..-D. Baumann (Hrsg.), 683-745.

KALVERKÄMPER, Hartwig/Klaus-Dieter BAUMANN (Hrsg.) (1996) Fachliche Textsorten: Komponenten - Relationen - Strategien. Tübingen: Gunter Narr.

KEIL, Denise (2007) Fachwissenstransfer als Einheit von Fachdenken, Fachwissen und Fachsprache: Dargestellt an deutschen und französischen Hochschullehrbüchern der Medizin. Hamburg: Dr. Kovac.

KLAUKE, Michael (1993) Instruktive Fachtexte des Englischen. Frankfurt a. Main: Peter Lang.

KLEIN, Josef/Ulla FIX (Hrsg.) (1997) Textbeziehungen: Linguistische und literaturwissenschaftliche Beiträge zur Intertextualität. Tübingen: Stauffenburg.

KLEIN, Josef (2000) „Intertextualität, Geltungsmodus, Texthandlungsmuster. Drei vernachlässigte Kategorien der Textsortenforschung - exemplifiziert an politischen und medialen Textsorten." In: K. Adamzik (Hrsg.), 31-44.

KNAPP, Karlfried (2004) „Interkulturelle Kommunikation“. In: K. Knapp et al. (Hrsg.), Angewandte Linguistik: Ein Lehrbuch. Tübingen/Basel: A. Francke, 409-430.

KNOBLAUCH, Hubert (2005) Wissenssoziologie. Konstanz: UVK Verlagsgesellschaft. 
KRAUSE, Wolf-Dieter (Hrsg.) (2000) Textsorten. Kommunikationslinguistische und konfrontative Aspekte. Frankfurt a. Main: Peter Lang.

KRISTEVA, Julia (1972) „Bachtin, das Wort, der Dialog und der Roman.“ In: J. Ihwe (Hrsg.), Literaturwissenschaft und Linguistik: Ergebnisse und Perspektiven. Bd. 3 (Zur linguistischen Basis der Literaturwissenschaft II). Frankfurt a. Main: Peter Lang, 345-375.

KÜHN, Andrea (2007) Kontrastive Denkstilanalyse deutscher und britischer Werke zur Geschichte des Dritten Reiches. Hamburg: Dr. Kovac.

LACHMANN, Renate (1990) Gedächtnis und Literatur. Intertextualität in der russischen Moderne. Frankfurt a. Main: Suhrkamp.

LÄPPLE, Dieter (1991) „Essay über den Raum: für ein gesellschaftswissenschaftliches Raumkonzept.“ In: H. Häussermann et al. (Hrsg.), Stadt und Raum. Pfaffenweiler: Centaurus Verlag, 157-207.

LAIKO, Alexej (2004) Intertextualität in der Übersetzung. Frankfurt a. Main: Peter Lang.

LEONT'EV, A. A./A. N. LEONT'EV/E. G. JUDIN (1984) Grundfrage einer Theorie der sprachlichen Tätigkeit. Berlin: Akademie Verlag.

MAYER, Felix (1998) Eintragsmodelle für terminologische Datenbanken: Ein Beitrag zur übersetzungsorientierten Terminographie. Tübingen: Gunter Narr.

MICHEL, Georg (Hrsg.) (1985) Grundfragen der Kommunikationsbefähigung. Leipzig: Bibliographisches Institut.

MORELLO, Christiane (2006) Die Textsorte Lebenslauf aus kontrastiver Sicht: Dargestellt am Deutschen und Französischen. Hamburg: Dr. Kovac.

MÜLLER, Andreas P. (2006) Sprache und Arbeit: Aspekte einer Ethnographie der Unternehmenskommunikation. Tübingen: Gunter Narr.

OSOLNIK KUNC, Viktorija (2006) Fachkommunikative Verständlichkeit in der Verwaltungssprache. Dargestellt am Deutschen und Slowenischen. Hamburg: Dr. Kovac.

OSTAPENKO, Valentyna (2007) Vernetzung von Fachtextsorten. Textsorten der Normung in der technischen Harmonisierung. Berlin: Frank \& Timme.

OPILOWSKI, Roman (2005) Intertextualität in der Werbung der Printmedien. Frankfurt a. Main: Peter Lang.

PAUEN, Michael/Gerhard ROTH (Hrsg.) (2001) Neurowissenschaften und Philosophie: Eine Einführung. München: Wilhelm Fink.

PETÖFI, Janos S./Terry OLIVI (1988) „Schöpferische Textinterpretation. Einige Aspekte der Intertextualität.“ In: J. S. Petöfi/T. Olivi (Hrsg.), Von der verbalen Kommunikation zur symbolischen Bedeutung. Hamburg: Helmut Buske, 335-350.

PREYER, Gerhard/Maria ULKAN/Alexander ULFIG (Hrsg.) (1997) Intention - Bedeutung - Kommunikation: Kognitive und handlungstheoretische Grundlagen der Sprachtheorie. Opladen: Westdeutscher Verlag.

RAASCH, Albert/Wolfgang KÜHLWEIN (Hrsg.) (1984) Btx Bildschirmtext. Tübingen: Gunter Narr.

RAJEWSKI, Irina O. (2002) Intermedialität. Tübingen/Basel: A. Francke. 
RICKHEIT, Gert/Hans STROHNER (1993) Grundlagen der kognitiven Sprachverarbeitung. Tübingen/Basel: Francke.

SCHÄFER, Daniela (2010) Kontrastive Analyse der Fachtextsorte ,Studentische Seminararbeit' aus Spanien und Deutschland. Hamburg: Dr. Kovac.

SCHIPPAN, Thea (1984) Lexikologie der deutschen Gegenwartssprache. Leipzig: Bibliographisches Institut.

SCHMIDT, Wilhelm (Hrsg.) (1981) Funktional-kommunikative Sprachbeschreibung. Leipzig: Bibliographisches Institut.

SCHRÖDER, Hartmut (Hrsg.) (1993) Fachtextpragmatik. Tübingen: Gunter Narr.

SCHRÖDER, Thomas (2003) Die Handlungsstruktur von Texten. Ein integrativer Beitrag zur Texttheorie. Tübingen: Gunter Narr.

SCHWEIGKOFLER, Anny (2007) Intertextualität beim Erwerb einer fremden Sprache. Hamburg: Dr. Kovac.

STICHWEH, Rudolf (2008) „Zur Soziologie des Weltereignisses.« In: St. Nacke/R. Unkelbach/T. Werron (Hrsg.), Weltereignisse. Theoretische und empirische Perspektiven. Wiesbaden: VS Verlag für Sozialwissenschaften, 17-40.

SWALES, John M. (2004) Research Genres: Exploration and Applications. Cambridge: University Press.

TAKAYAMA-WICHTER, Taeko (2005) „Das Comic-Element beim Wissenstransfer - sein Stellenwert in der japanischen Gesellschaft und seine Intertextualität." In: S. Wichter/G. Antos, Wissenstransfer zwischen Experten und Laien. Umriss einer Transferwissenschaft. Frankfurt a. Main: Peter Lang, 203-230.

THOMAS, Alexander (2000) „Forschungen zur Handlungswirksamkeit von Kulturstandards." Handlung, Kultur, Interpretation - Zeitschrift für Sozial- und Kulturwissenschaften 9, 2, 231-279.

TIMM, Christian (1996) „Das Vorwort - eine ,Textsorte-in-Relation'““ In: H. Kalverkämper/K.-D. Baumann (Hrsg.), 458-467.

VIRILIO, Paul (1986) Speed and Politics: An Essay on Dromology. Taschenbuch Autonomedia.

VIRILIO, Paul (2008) Ästhetik des Verschwindens. Berlin: Merve Verlag.

VOGLER, Daniela (2006) Denkstile in der naturwissenschaftlich-technischen Fachkommunikation. Hamburg: Dr. Kovac.

VOGLER, Daniela (2008) Der technikwissenschaftliche Denkstil in seiner sprachlichen Manifestation: Dargestellt am Beispiel der Werkstoffwissenschaft. Dissertation. Hamburg: Dr. Kovac.

VOGLER, Daniela (2009) „Der technikwissenschaftliche Denkstil in seiner sprachlichen Manifestation. Am Beispiel der referentiellen Intertextualität in werkstoffwissenschaftlichen Zeitschriftenartikeln.“ In: K.-D. Baumann (Hrsg.) (2009a), 223-256.

WEIGEL, Victoria Freya (2013) Uniforme und differente topologische Modelle im Vergleich. München/Ravensburg: GRIN Verlag.

WICHTER, Sigurd/Gerd ANTOS (2001) Wissenstransfer zwischen Experten und Laien. Umriss einer Transferwissenschaft. Frankfurt a. Main: Peter Lang. 
WIESE, Ingrid (2004) „Texte des ,informierten Laien“ aus fachsprachenlinguistischer Sicht.“ In: K.-D. Baumann/H. Kalverkämper (Hrsg.), Pluralität in der Fachsprachenforschung. Tübingen: Gunter Narr, 121-127.

WILDGEN, Wolfgang (2008) Kognitive Grammatik. Klassische Paradigmen und neue Perspektiven. Berlin/New York: de Gruyter.

WILSKE, Ludwig/Wolf-Dieter KRAUSE (1987) „Intertextualität als allgemeine und spezielle Texteigenschaft. «Wissenschaftliche Zeitschrift der Pädagogischen Hochschule Potsdam 5, 890-895.

YLÖNEN, Sabine (2001) Entwicklung von Textsortenkonventionen. Frankfurt a. Main: Peter Lang.

\section{Zusammenfassung}

FACHTEXTE-IN-VERNETZUNG ALS INTERDISZIPLINÄRER ZUGANG ZU FACHLICHEN KOMMUNIKATIONSRÄUMEN - EIN NEUES PARADIGMA DER AKTUELLEN FACHKOMMUNIKATIONSFORSCHUNG

Die vorliegende Betrachtung von Fachtexten-in-Vernetzung und Kommunikationsräumen hat deutlich gemacht, dass zwischen beiden ein außerordentlich komplexes Zusammenspiel besteht. Die in der Fachkommunikation zu beobachtenden Vertextungsregularitäten führen zu bestimmten Fachtext(-sort)en in-Vernetzung, die als mehrschichtiger Zugang zu fachlichen Kommunikationsräumen fungieren. Diese bestehen aus einem vielschichtigen Beziehungsgeflecht von Dimensionen der Kulturen, Sozialität, Situativität, Intermedialität, des Fachdenkens, Fachinhalts, der Funktion, Textstrukturen, Syntax und Morphologie, des Stils, der Lexik und Semantik, der Grafik, Orthographie, Phonetik und Phonologie.

Zukünftigen Untersuchungen bleibt es vorbehalten, das skizzierte interdisziplinäre Modell der Fachtexte-in-Vernetzung als fachliche Kommunikationsräume durch empirische Analysen auf den einzelnen Bezugsebenen weiter zu untermauern.

Schlüsselwörter: Fachtexte-in-Vernetzung, Vertextungsregularitäten, Intertextualität, Kommunikationsräume, Dimensionen der Kommunikationsräume

\section{Abstract \\ LSP TEXT NETWORKS AS AN INTERDISCIPLINARY APPROACH TO LSP COMMUNICATION SPACES - A NEW PARADIGM FOR THE LATEST LSP COMMUNICATION RESEARCH}

One focus of interdisciplinary LSP ${ }^{3}$ text research lies in illustrating LSP intertextuality leading to specific LSP text networks. These are closely linked to the formation

3 LSP - Language For Specific Purposes/Fachsprache. 
of specialist communication spaces where (non-)experts interact with each other, exchanging LSP text networks and coordinating socio-economic activities at different levels of technicality.

Communication spaces are necessarily shaped by different factors: culture, sociality, situativity, intermediality, thinking style, scientific subject, functionality, text structure, syntax and morphology, style, lexis and semantics, graphics, orthography, phonetics and phonology.

Our recent interdisciplinary empirical analyses have provided a number of new insights, some of which may prove to be particularly significant for further interdisciplinary LSP text networks research.

Key words: LSP text networks, text-processing principles, intertextuality communication spaces, dimensions of communication spaces

\section{Povzetek \\ STROKOVNA BESEDILA V OMREŽJIH \\ KOT INTERDISCIPLINARNI PRISTOP H KOMUNIKACIJSKIM PROSTOROM \\ STROKE - NOVA PARADIGMA AKTUALNEGA RAZISKOVANJA STROKOVNE KOMUNIKACIJE}

Obravnava strokovnih besedil $\mathrm{v}$ omrežjih ter komunikacijskih prostorov je jasno pokazala, da obstaja med njimi izredno kompleksno prepletanje. Zakonitosti tvorjenja besedil, ki jih je mogoče opazovati v strokovni komunikaciji, vodijo do določenih besedilnih vrst v omrežjih, ki delujejo kot večplasten pristop do komunikacijskih prostorov stroke. Te prostore sestavljajo večplastna razmerja med dimenzijami kultur, družbenih okoliščin, situacijskosti, intermedialnosti, strokovnega razmišljanja, strokovnih vsebin, funkcij, besedilnih struktur, skladnje in morfologije, sloga, leksike in semantike, grafike, pravopisa, fonetike in fonologije. Skicirani interdisciplinarni model strokovnih besedil $\mathrm{v}$ omrežjih bo $\mathrm{v}$ prihodnje zagotovo mogoče $\mathrm{z}$ empiričnimi analizami na posameznih ravninah prikazati kot komunikacijske prostore stroke.

Ključne besede: strokovna besedila $\mathrm{v}$ omrežjih, zakonitosti tvorjenja besedil, medbesedilnost, komunikacijski prostori, dimenzije komunikacijskih prostorov 